\title{
ASTCT, CIBMTR, and EBMT clinical practice recommendations for transplant and cellular therapies in mantle cell lymphoma
}

\author{
Pashna N. Munshi $\mathbb{D}^{1} \cdot$ Mehdi Hamadani $\mathbb{(}^{2}$ - Ambuj Kumar $\mathbb{D}^{3}$ - Peter Dreger $\mathbb{D}^{4}$ - Jonathan W. Friedberg ${ }^{5}$. \\ Martin Dreyling ${ }^{6} \cdot$ Brad Kahl $^{7} \cdot$ Mats Jerkeman $\mathbb{D}^{8} \cdot$ Mohamed A. Kharfan-Dabaja $\mathbb{D}^{9} \cdot$ Frederick L. Locke $\mathbb{D}^{10}$. \\ Mazyar Shadman ${ }^{11}$ - Brian T. Hill ${ }^{12}$ - Sairah Ahmed $\mathbb{D}^{13} \cdot$ Alex F. Herrera $^{14} \cdot$ Craig S. Sauter $^{15}$. \\ Veronika Bachanova ${ }^{16}$. Nilanjan Ghosh ${ }^{17}$. Matthew Lunning ${ }^{18} \cdot$ Vaishalee P. Kenkre $^{19}$ - Mahmoud Aljurf ${ }^{20}$. \\ Michael Wang $\mathbb{D}^{21} \cdot$ Kami J. Maddocks ${ }^{22}$ - John P. Leonard ${ }^{23} \cdot$ Manali Kamdar $^{24}$ - Tycel Phillips ${ }^{25}$. \\ Amanda F. Cashen ${ }^{26}$. David J. Inwards ${ }^{27}$ - Anna Sureda ${ }^{28}$. Jonathon B. Cohen ${ }^{29}$. Sonali M. Smith ${ }^{30}$. \\ Carmello Carlo-Stella $\mathbb{1}^{31} \cdot$ Bipin Savani ${ }^{32} \cdot$ Stephen P. Robinson ${ }^{33} \cdot$ Timothy S. Fenske $^{34}$
}

Received: 4 March 2021 / Revised: 8 March 2021 / Accepted: 25 March 2021 / Published online: 20 August 2021

(c) The Author(s) 2021

\begin{abstract}
Autologous (auto-) or allogeneic (allo-) hematopoietic cell transplantation (HCT) are accepted treatment modalities for mantle cell lymphoma (MCL). Recently, chimeric antigen receptor (CAR) T-cell therapy received approval for MCL; however, its exact place and sequence in relation to HCT is unclear. The ASTCT, CIBMTR, and the EBMT, jointly convened an expert panel to formulate consensus recommendations for role, timing, and sequencing of auto-, allo-HCT, and CAR T-cell therapy for patients with newly diagnosed and relapsed/refractory (R/R) MCL. The RAND-modified Delphi method was used to generate consensus statements. Seventeen consensus statements were generated; in the first-line setting auto-HCT consolidation represents standard-of-care in eligible patients, whereas there is no clear role of allo-HCT or CAR T-cell therapy, outside of a clinical trial. In the R/R setting, the preferential option is CAR T-cell therapy especially in MCL failing or intolerant to at least one Bruton's tyrosine kinase inhibitor, while allo-HCT is recommended if CAR T-cell therapy has failed or is not feasible. In the absence of contemporary evidence-based data, the panel found RAND-modified Delphi methodology effective in providing a formal framework for developing consensus recommendations for the timing and sequence of cellular therapies for MCL.
\end{abstract}

\section{Introduction}

Mantle cell lymphoma (MCL) is a B-cell lymphoma which displays significant clinical and molecular heterogeneity [1]. In most cases, it follows an aggressive clinical course; however, a subset of patients can have indolent disease [2].

This article is co-published in the journals Bone Marrow Transplantation and Transplantation and Cellular Therapy https://doi. org/10.1038/s41409-021-01288-9 or https://doi.org/10.1016/j.jtct. 2021.03 .001 .

Supplementary information The online version contains supplementary material available at https://doi.org/10.1038/s41409021-01288-9.

Mehdi Hamadani

mhamadani@mcw.edu

Extended author information available on the last page of the article
Similarly, the management of MCL varies greatly in clinical practice within the United States and abroad [3, 4]. This variability applies to both the frontline as well as the relapsed/refractory (R/R) settings [5]. Pertaining to frontline therapy, some advocate induction (chemoimmuno-) therapies followed high dose therapy (HDT) and autologous hematopoietic cell transplant (auto-HCT) consolidation, while others prefer combination chemoimmunotherapy regimens alone without subsequent HDT consolidation. Treatment strategies are even more discordant if high-risk features are present, such as TP53 alterations, and a high proliferation index [6-8]. In the $\mathrm{R} / \mathrm{R}$ setting, there is also variability in practice, made even more complex by the advent of newer treatment modalities such as Bruton's tyrosine kinase (BTK) inhibitors [9], lenalidomide, and chimeric antigen receptor (CAR) T-cell therapy [10].

Auto-HCT consolidation has been used for over 20 years in the management of MCL patients [11, 12], and is 
associated with improved progression-free survival and potentially overall survival (OS) following conventional chemoimmunotherapy in both prospective and retrospective studies [11-14]. However, it is less clear whether auto-HCT has a benefit following more intensive chemoimmunotherapy, such as R-Hyper-CVAD/cytarabine/methotrexate [15]. Allo-HCT is a potentially curative modality for MCL $[12,16]$. However, with a 1-year treatment related mortality ranging from 10 to $20 \%$, and the additional risk of chronic graft-versus-host disease, allo-HCT has generally been reserved for the R/R setting [3, 17].

There have been recent important advances in MCL therapy including the demonstration of a survival benefit with rituximab maintenance following auto-HCT [18], the advent of first and second-generation BTK inhibitors for R/ R MCL [19], and the recent approval of the first commercially available CAR T-cell therapy (brexucabtagene autoleucel) for MCL [10]. In the case of brexucabtagene autoleucel, the objective and complete response rates were $93 \%$ and $67 \%$, respectively, with durable remission for more than half of patients. Importantly, although this registration trial exclusively studies MCL patients who had been previously treated with BTK inhibitor therapy, U.S. regulatory approval provides an indication for all R/R MCL regardless of prior exposure to BTK inhibitor. Given these novel treatment options, guidance on the contemporary role, optimal timing, and sequencing of cellular therapies in MCL is warranted. Clinical practice recommendations addressing areas of clinical ambiguity can aid not only the treating transplant and cellular therapy physicians but can also inform lymphoma experts' and community hematologists' practice for referring these patients to transplant and cell therapy programs. The American Society of Transplantation and Cellular Therapy (ASTCT), Center of International Blood and Marrow Transplant Research (CIBMTR), and the European Society for Blood and Marrow Transplantation (EBMT), therefore undertook a joint project to formulate consensus recommendations regarding the role, timing and sequencing of auto-, allo-HCT and CAR T-cell therapy for patients with newly diagnosed and $\mathrm{R} / \mathrm{R}$ MCL.

\section{Methods}

\section{Panel composition}

The development of practice recommendations was approved by ASTCT, CIBMTR, and EBMT, the three leading international organizations in the field of HCT and cellular therapies. As an initial step, a steering committee was formed comprising of six members including two project leaders/coordinators, one representative each from
ASTCT, EBMT, CIBMTR, and an independent methodologist with expertise in systematic reviews, meta-analysis, and the RAND-modified Delphi method. The steering committee was responsible for drafting the protocol, initial draft of consensus statements based on clinical expertise, clinical practice considerations and setting up of the expert panel [20]. The aim was to put together an expert panel with a balanced distribution of 'MCL' and 'cellular therapy and transplant' experts, in order to have broad expertise and to cover a wide spectrum of views, whilst keeping administrative efforts manageable as previously recommended $[21,22]$. The panel of experts consisted of physicians with diverse geographical representation and expertise in the field, as demonstrated by their track-record of peerreviewed publications, leadership on clinical trials relevant to the consensus project and by their involvement in national and international lymphoma or transplant organizations. Additionally, a physician representing a community-based practice was included in the panel, as previously recommended (NG) [20]. The final consensus panel consisted of 33 physicians and investigators, including members of the steering committee $(n=5)$, except the (non-clinical) independent methodologist, who did not vote on the recommendations (AK).

\section{Consensus methodology $[20,21]$}

The RAND-modified Delphi method was utilized to generate consensus statements addressing the role, timing, and sequence of HCT and CAR T-cell therapies in patients with newly diagnosed and R/R MCL. In the Delphi method, the participants rate the statements anonymously in at least two rounds of evaluations. In the modified version of the method, a face-to-face meeting with presentation of the results precedes the second round of rating [20-22]. Due to the ongoing COVID-19 pandemic, virtual (ZOOM, San Jose, CA) platform was used in lieu of a face-to-face meeting. Details regarding the systematic step-by-step approach used in this project, are illustrated in Table 1.

After the panel selection, a Baseline Demographics and Scope $(B D \& S)$ survey was developed to determine the scope of the project. Participants were invited to submit their suggestions regarding the scope of the consensus project and provide input about the clinical issues relevant to clinical practice (details in Supplementary Appendix). After finalization of the scope of the consensus project, the steering committee formulated preliminary consensus statements based on expert opinion for first round of voting (details in Supplementary Appendix; Tables 1S, 2S).

The First Voting Survey included 19 consensus statements. Panel members rated each statement electronically. The steering committee methodologist analyzed and summarized the results, while keeping the individual ratings 


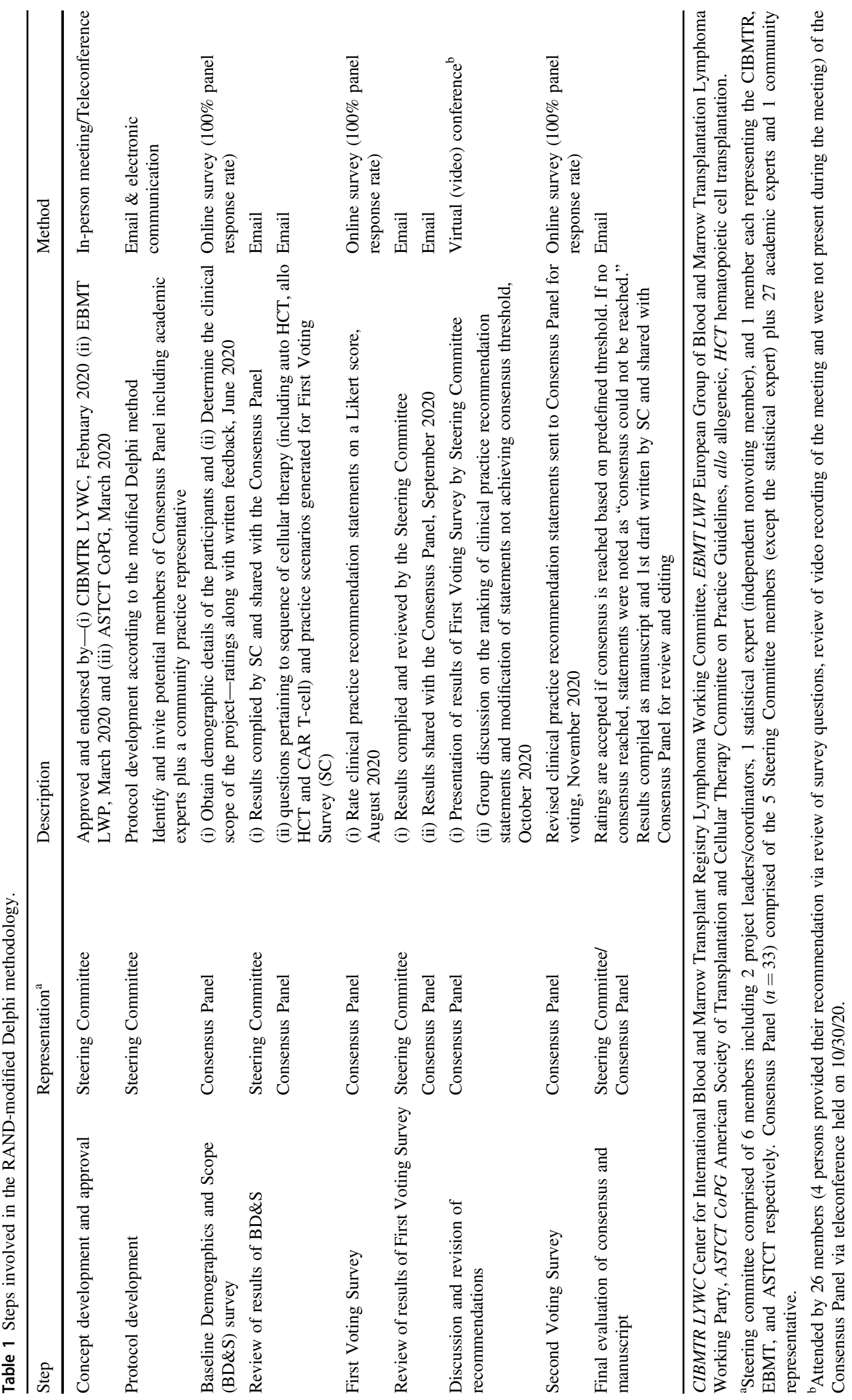


anonymous. A specific statement was defined as having achieved formal consensus, if $\geq 75 \%$ of the panel members voted to agree with the proposed statement. The results of First Voting Survey, along with the statements not reaching the threshold of consensus were presented at the virtual teleconference of the panel members. Consensus statements that met the predefined criteria for formal consensus were recommended for approval. Statements that failed to achieve predefined criteria for consensus were discussed during the virtual meeting and based on the discussions the statements were modified for re-voting or dropped. The Second Voting Survey was sent to all the panel members for rating of the reformulated or newly added statements.

All surveys were administered online using www.Qua ltrics.com (Qualtrics LLC, Provo, UT, USA) and results were reviewed and collated independently by the methodological expert. At each step of the process, the electronic survey also allowed the participating members to provide written feedback and comments about each statement. Collated results were shared via email with the consensus panel members in real time after each step was completed to ensure transparency of the process. The final consensus statements were graded based on the strength and level of supporting evidence, according to the Agency of Healthcare Research and Quality (AHRQ) grading [23].

\section{Results}

\section{Member participation}

Table 2 describes the baseline characteristics of consensus panel members. Included were transplant and cell-therapy physicians ( $>75 \%$ of practice time in HCT), non-cell therapy academic physicians, mixed practitioners and a community-based practitioner. A mixed practice was defined as practitioners devoting $\sim 50 \%$ of clinical time to HCT and non-cell therapy related lymphoma, each. In general, panelist participation and response rates were excellent. During the voting process, $100 \%(n=33)$ panel member participation was noted for the $B D \& S$, First Voting and Second Voting surveys. The virtual meeting was attended by 26 members including 4 members who provided their absentee vote by providing written feedback in advance $(n=1)$ or following the meeting, after reviewing the video recordings of the teleconference $(n=3)$.

\section{First Voting Survey}

The First Voting survey consisted of 19 statements specific to the role of auto-HCT in eligible newly diagnosed MCL patients (6 statements) and R/R MCL patients (2 statements), allo-HCT for newly diagnosed MCL patients
Table 2 Demographic information of members of Consensus Panel.

\begin{tabular}{lc}
\hline Member demographics & $N=33^{\mathrm{a}}(\%)$ \\
\hline Gender & $24(72.7 \%)$ \\
Male & $9(27.3 \%)$ \\
Female & \\
Setting of practice & $31(93.9 \%)$ \\
Academic & $2(6.1 \%)$ \\
Community & $22(66.7 \%)$ \\
Years of clinical experience in lymphoma and/or HCT & practice \\
$>10$ & $9(27.3 \%)$ \\
$6-10$ & $2(6.0 \%)$ \\
$\leq 5$ & \\
Description of clinical practice & $3(9.0 \%)$ \\
Non-transplant lymphoma practice & $5(15.2 \%)$ \\
Primarily HCT and/or cell therapy practice & $25(75.8 \%)$ \\
Combined lymphoma and HCT/cell therapy practice & \\
Region of practice & \\
North America & $26(78.8 \%)$ \\
Europe & $6(18.2 \%)$ \\
Middle East & $1(3.0 \%)$
\end{tabular}

Estimated number of newly diagnosed lymphoma patients seen by individual member annually

$\begin{array}{lc}>75 & 23(69.7 \%) \\ 51-75 & 6(18.2 \%) \\ 26-50 & 3(9.1 \%) \\ \leq 25 & 1(3.0 \%)\end{array}$

Estimated number of mantle cell lymphoma patients seen by individual member annually

$\begin{array}{lc}>40 & 6(18.2 \%) \\ 31-40 & 3(9.1 \%) \\ 21-30 & 14(42.4 \%) \\ \leq 20 & 10(30.3 \%)\end{array}$

Estimated annual transplant volume at respective programs (number of autologous plus allogeneic HCT)

$\begin{array}{lc}>300 & 13(39.4 \%) \\ 201-300 & 7(21.2 \%) \\ 101-200 & 9(27.3 \%) \\ 51-100 & 3(9.1 \%) \\ \leq 50 & 1(3.0 \%)\end{array}$

Estimated annual autologous HCT performed at respective centers

$\begin{array}{ll}>250 & 7(21.2 \%) \\ 201-250 & 3(9.1 \%) \\ 151-200 & 5(15.2 \%) \\ 101-150 & 8(24.2 \%) \\ 51-100 & 8(24.2 \%) \\ \leq 50 & 2(6.1 \%)\end{array}$

Estimated annual autologous HCT performed at respective centers for lymphoma (Hodgkin plus non-Hodgkin)

$>200$

$1(3.0 \%)$

$151-200$

$3(9.1 \%)$ 
Table 2 (continued)

\begin{tabular}{lc}
\hline Member demographics & $N=33^{\mathrm{a}}(\%)$ \\
\hline $101-150$ & $2(6.1 \%)$ \\
$51-100$ & $10(30.3 \%)$ \\
$26-50$ & $13(39.4 \%)$ \\
$\leq 25$ & $4(12.1 \%)$
\end{tabular}

Estimated annual CAR T-cell therapies performed at respective centers for lymphoma (on or off clinical trial)

\begin{tabular}{lc}
$>20$ & $21(63.6 \%)$ \\
$16-20$ & $3(9.1 \%)$ \\
$11-15$ & $5(15.2 \%)$ \\
$\leq 10$ & $4(12.1 \%)$ \\
\hline
\end{tabular}

HCT hematopoietic cell transplantation, $C A R$ chimeric antigen receptor.

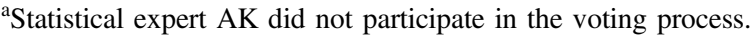

${ }^{\mathrm{b}}$ Countries represented include: United States $=26$, Germany $=2$, United Kingdom $=1$, Spain $=1$, Italy $=1$, Sweden $=1$, Saudi Arabia $=1$.

(3 statements) and allo-HCT and/or CAR T-cell therapy for R/R MCL (8 statements). All but five statements achieved consensus by predefined criteria (Table 1S). The results of the First Voting Survey were electronically shared with all panel members. The five statements not achieving consensus $(<75 \%$ agreement) during the prior voting process were reviewed by the steering committee and presented to the panel members at the virtual video conference. The ensuing discussion resulted in one statement regarding autoHCT being abandoned and all other statements being revised. In total three statements were proposed (reformulated statements $=2$, merged statement $=1$ ) for the Second Voting Survey. Table $2 \mathrm{~S}$ shows outcomes of the virtual video conference.

\section{Second Voting Survey}

All statements included in the Second Voting Survey (reformulated statements $=2$, merged statement $=1$ ), met the predefined criteria for consensus (Table $2 \mathrm{~S}$ ). The final consensus recommendations on auto-HCT, allogeneic HCT and CAR T-cell therapy for upfront and relapsed MCL consisting of 17 consensus statements are shown in Tables 3 and 4 .

\section{Discussion}

In this project, a broadly representative panel of lymphoma, transplant, and cellular therapy experts with diverse practice experience and geographical representation, endorsed by ASTCT, EBMT, and CIBMTR, was formed to provide consensus recommendations on the role of auto-HCT, allo-
HCT, and CAR T-cell therapy for newly diagnosed and R/R MCL. Considering the limitations in existing data for treatments with cellular immunotherapy for MCL and the recently approved CAR T-cell therapy (brexucabtagene autoleucel) [10] for relapsed refractory MCL, the optimal sequencing of these treatments in the era of other novel therapies like BTK inhibitors is unknown. Therefore, this undertaking was conceived to provide a rational basis for clinical guidance where evidence is limited and resulted in seventeen consensus recommendations.

\section{Recommendations in the front-line setting without TP53 aberrations}

Seven consensus statements were generated for transplantation and CAR T-cell treatments in the frontline setting for MCL (Table 3). Taking into account the European MCL Network randomized study for upfront auto-HCT consolidation in MCL $[4,13]$ and several other historical prospective trials [18, 24-26], the panel recommended autoHCT as consolidation therapy in eligible, newly diagnosed MCL patients (without TP53 mutation or bi-allelic deletion) in complete remission or partial remission after first-line therapies (Grade A recommendation, Table 3, \#1). While this is in keeping with current guidelines [3], the consensus panel does acknowledge, that owing to the lack of evidence for a survival benefit with upfront auto-HCT consolidation, some experts and centers do not routinely recommend this modality after front line intensive induction regimens. In addition, the panel did not recommend auto-HCT as consolidative therapy in MCL patients with disease refractory (or unresponsive) to most recent line of therapy (Grade B recommendation, Table 3, \#2). Finally, given the lack of prospective data to guide consolidative auto-HCT based on the presence (or absence) of measurable residual disease (MRD), the panel did not recommend using MRD testing to determine whether auto-HCT consolidation should be applied, outside of a clinical trial (Grade $\mathrm{C}$ recommendation, Table 3, \#3). The ongoing US Intergroup phase III study (ECOG-ACRIN 4151; NCT03267433) is randomizing MRD-negative MCL patients to undergo auto-HCT followed by maintenance rituximab or maintenance rituximab alone to bridge this knowledge gap.

The panel was not able to reach consensus on recommending collection and storage of peripheral blood hematopoietic progenitor cells (HPC) for patients not undergoing an upfront auto-HCT (Tables $2 \mathrm{~S}$ and $3 \mathrm{~S}$ ). The cost of collecting and storing HPC for future use [27] and ambiguity about the role of auto-HCT in R/R setting in the CAR T-cell era, were among the major concerns raised by the expert panel. In addition, it was felt that, if necessary, peripheral blood HPC collection is feasible at a later point in the disease process. Although there are a number of prognostic 
Table 3 Final clinical practice guidelines consensus statements for transplantation and CAR T-cell treatments in the front-line setting for mantle cell lymphoma.

\begin{tabular}{|c|c|c|}
\hline Consensus statements & $\begin{array}{l}\text { Grading of } \\
\text { recommendations }^{\mathrm{a}}\end{array}$ & $\begin{array}{l}\text { Percentage of } \\
\text { panelists in } \\
\text { agreement }\end{array}$ \\
\hline $\begin{array}{l}\text { 1. The panel recommends autologous HCT as consolidation therapy in eligible, newly diagnosed } \\
\text { MCL patients (without TP53 mutation or bi-allelic deletion) in complete remission or partial } \\
\text { remission after first-line therapies. }\end{array}$ & A & $87.9 \%$ \\
\hline $\begin{array}{l}\text { 2. The panel does not recommend autologous transplantation as consolidation therapy in MCL } \\
\text { patients with disease not responsive to most recent anti-lymphoma therapy. }\end{array}$ & B & $100 \%$ \\
\hline $\begin{array}{l}\text { 3. The panel does not recommend using measurable residual disease (MRD) testing to guide use of } \\
\text { autologous transplant consolidation after first-line therapies in MCL, outside the setting of a } \\
\text { clinical trial. }\end{array}$ & $\mathrm{C}$ & $100 \%$ \\
\hline $\begin{array}{l}\text { 4. The panel does not recommend using MIPI or MIPI-c prognostic score as a criterion determining } \\
\text { use of autologous transplantation as consolidation therapy in eligible newly diagnosed MCL } \\
\text { patients in first complete remission or partial remission after first-line therapies. }\end{array}$ & $\mathrm{C}$ & $100 \%$ \\
\hline $\begin{array}{l}\text { 5. The panel does not recommend allogeneic transplant consolidation in MCL patients (without } \\
\text { TP53 mutation or bi-allelic deletion), achieving a complete or partial remission after first-line } \\
\text { therapies. }\end{array}$ & B & $97 \%$ \\
\hline $\begin{array}{l}\text { 6. The panel does not recommend consolidation with CAR T-cell therapy in MCL patients, } \\
\text { achieving a complete or partial remission after first-line therapies, outside the setting of a } \\
\text { clinical trial. }\end{array}$ & $\mathrm{C}$ & $100 \%$ \\
\hline $\begin{array}{l}\text { 7. If a TP53 mutation (or bi-allelic deletion) is present, the panel recognizes that outcomes are poor } \\
\text { for MCL patients in complete or partial remission after first-line therapies who then undergo } \\
\text { autologous transplantation. However, no specific alternative strategy has yet been shown to } \\
\text { improve outcomes in such patients. Therefore, the panel recommends considering autologous } \\
\text { transplant consolidation as well as alternative consolidation strategies (e.g. CAR T-cell therapy or } \\
\text { allogeneic transplantation), ideally in the context of a clinical trial, for such patients. }\end{array}$ & $\mathrm{C}$ & $100 \%$ \\
\hline
\end{tabular}

$H C T$ hematopoietic cell transplantation, PET/CT positron emission tomography/computed tomography, $M C L$ mantle cell lymphoma.

${ }^{a}$ Agency of Healthcare Research and Quality (AHRQ) grading of recommendations based on level of evidence [23]:

$\mathrm{A}=$ There is good research-based evidence to support the recommendation;

$\mathrm{B}=$ There is fair research-based evidence to support the recommendation;

$\mathrm{C}=$ The recommendation is based on expert opinion and panel consensus:

$\mathrm{X}=$ There is evidence of harm from this intervention.

factors that can be used to predict outcomes for MCL, such as the MCL International Prognostic Index (MIPI) score $[24,28,29]$, owing to the lack of supportive data the panel did not recommend using MIPI prognostic score as a criterion for selecting patients for auto-HCT as consolidation therapy (Grade $\mathrm{C}$ recommendation, Table 3, \#4).

While the panel anticipates that future trials will investigate the role of CAR T-cell therapies as consolidation following frontline treatment, it does not recommend this approach, outside the setting of a clinical trial (Grade C recommendation, Table 3, \#6).

\section{Recommendations in the front-line setting with TP53 aberrations}

The panel recognized that outcomes of MCL patients with TP53 mutation (or bi-allelic deletion) who are in complete or partial remission after first-line treatments are poor following auto-HCT consolidation [6]. Although there is preliminary evidence that both allo-HCT and CAR T-cell therapies may overcome treatment resistance conferred by TP53 aberrations $[10,30]$ no alternative strategies have been shown to improve outcomes of first-line therapy in such patients in a randomized trial. Therefore, the panel cautiously recommended considering auto-HCT consolidation as well as other alternative consolidation strategies (e.g. CAR T-cell therapy or allo-HCT), ideally in the context of a clinical trial, for such patients (Grade $\mathrm{C}$ recommendation, Table 3, \#7).

\section{Recommendations in the relapsed/refractory setting}

The panel acknowledges that in the modern era of novel immunotherapies, auto-HCT will likely play a limited role in the management of $\mathrm{R} / \mathrm{R} \mathrm{MCL}$, particularly in the presence of TP53 aberrations where the panel does not recommend auto-HCT (Grade B recommendations, Table 4, \#1). However, among standard risk MCL patients (e.g. those lacking a TP53 mutation or bi-allelic deletion) not having undergone auto-HCT in first remission, the panel felt considering HDT consolidation therapy in the subset of 
Table 4 Final clinical practice guidelines consensus statements for transplantation and CAR T-cell treatments for relapsed and/or refractory mantle cell lymphoma.

\begin{tabular}{lll}
\hline Consensus statements & $\begin{array}{l}\text { Grading of } \\
\text { recommendations }^{\mathrm{a}}\end{array}$ & $\begin{array}{l}\text { Percentage of } \\
\text { panelists in } \\
\text { agreement }\end{array}$
\end{tabular}

1. If a TP53 mutation (or bi-allelic deletion) is present, the panel does not recommend autologous B $100 \%$ transplantation in relapsed MCL patients achieving a complete or partial remission after second or subsequent lines of therapy.

2. The panel recommends both CAR T-cell therapy or allogeneic transplant consolidation as C acceptable options, in relapsed MCL patients with TP53 mutation (or bi-allelic deletion) in a complete or partial remission after second or subsequent lines of therapy.

3. If a TP53 mutation (or bi-allelic deletion) is present, the panel recommends treatment with CAR B T-cells in relapsed MCL patients, with disease unresponsive to last anti-lymphoma therapy.

4. In relapsed MCL patients, the panel recommends offering CAR T-cell therapy before proceeding C $81.8 \%$ with allogeneic transplantation.

5. Regarding timing of CAR T-cell application in relapsed MCL patients (without TP53 mutation or B bi-allelic deletion), the panel recommends offering CAR T-cell therapy to patients relapsing after (or who are intolerant to) at least one BTK inhibitor.

6. The panel does not recommend allogeneic transplantation in relapsed MCL patients with disease B refractory to most recent anti-lymphoma treatment.

7 The panel recommends allogeneic transplantation for eligible relapsed MCL patients that have B achieved only a partial remission with a BTK inhibitor in second or subsequent treatment line, particularly in regions without access to CAR T-cell therapy or in subjects where such therapy is not feasible.

8. The panel recommends allogeneic transplantation in eligible MCL patients relapsing/progressing C after CAR T-cell therapy, if they achieve a complete or partial remission or if they have stable disease with subsequent anti-lymphoma therapies.

9. Among eligible MCL patients lacking a TP53 mutation (or bi-allelic deletion) not undergoing B autologous transplant consolidation following first-line therapies, the panel recommends considering autologous transplantation consolidation therapy in patients who have achieved a complete remission after second line chemo-immunotherapies.

10. The panel recommends considering allogeneic transplant consolidation in eligible MCL patients C who still have detectable disease, at 3 or more months following CAR T-cell therapy.

HCT hematopoietic cell transplantation, PET/CT positron emission tomography/computed tomography, $M C L$ mantle cell lymphoma.

${ }^{a}$ Agency of Healthcare Research and Quality (AHRQ) grading of recommendations based on level of evidence [23]:

$\mathrm{A}=$ There is good research-based evidence to support the recommendation;

$\mathrm{B}=$ There is fair research-based evidence to support the recommendation;

$\mathrm{C}=$ The recommendation is based on expert opinion and panel consensus:

$\mathrm{X}=$ There is evidence of harm from this intervention.

patients who have achieved a complete remission after second line chemo-immunotherapies particularly after long first remission, was reasonable, and supported by observations in more recent registry and other retrospective studies (Grade B recommendation, Table 4, \#9) [12].

Brexucabtagene autoleucel was FDA approved on July 24th, 2020 for the treatment of R/R MCL, prior to the voting survey \#1. The panel felt that the FDA label did not identify optimal timing of CAR T-cell therapy in R/R MCL. Considering the cost of this modality and the availability of other active targeted therapy options, the panel recommended that CAR T-cell therapy is best applied in $\mathrm{R} / \mathrm{R}$ MCL patients intolerant to, or relapsing after at least one
BTK inhibitor (Grade B recommendation, Table 4, \#5). This appears to be in accordance with the European label for brexucabtagene autoleucel, which was granted by the European Medicines Agency (EMA) in December 2020 (after the completion of this consensus project) and approved this CAR T-cell therapy for R/R MCL after two lines of systemic therapy including a BTKi. However, owing to the preliminary evidence of activity of CAR T-cell therapy in patients with TP53 mutation [10], application of CAR T-cell therapy as a second-line therapy (i.e. even without prior BTKi exposure) may be considered for such patients (Grade B recommendation, Table 4, \#3).

With the approval of CAR T-cell therapy for R/R MCL, the role of allo-HCT deserves re-evaluation. The panel 
recognizes the increased toxicities and life-threatening complications of allo-HCT, and therefore recommends considering CAR T-cell treatments prior to allo-HCT. In practical terms and taking into account recommendation \#5 (Table 4) for $\mathrm{R} / \mathrm{R}$ disease, this means that the treatment sequence would be to treat with BTK inhibitors until failure or intolerance, then move to CAR T-cell therapy, and reserve allo-HCT for CAR T-cell therapy failure. However, given the lack of comparative data of CAR T-cell therapy vs allo-HCT, the panel acknowledges that this recommendation represents expert opinion for clinicians to consider (Grade $\mathrm{C}$ recommendation, Table 4, \#4). Thus, allo-HCT remains an option as part of second-line treatment in eligible patients who achieve only a partial response to BTK inhibitors (which will be the majority of BTK inhibitor responders $[9,31])$, in particular in areas where CAR T-cell therapies are not available (Grade B recommendation, Table 4, \#7).

In addition, the panel considered allo-HCT a reasonable treatment option in R/R MCL patients who have relapsed after CAR T-cell therapy, particularly if the disease remains sensitive to subsequent treatment attempts (Grade C recommendation, Table 4, \#8) [16, 17, 32-34]. This recommendation can also be considered for those patients with persistent yet not progressive disease detectable beyond 3 months following CAR T-cell administration (Grade $\mathrm{C}$ recommendation, Table 4, \#10), taking into account the low probability of durable disease control in this subset [10].

\section{Conclusion}

In clinical scenarios where data from prospective studies are either scarce or unavailable, or in situations where therapeutic advances or new drug indications make patient populations included in published trials less relevant to contemporary clinical practice, formal consensus recommendations can be an invaluable resource in informing clinical decision making [35]. Expert opinions and recommendations in the form of review articles and treatment guidelines, while useful, lack methodological clarity and may be subject to bias [35]. In contrast, formulation of expert recommendations using established approaches, such as the RAND-modified Dephi method [20], provides a formal, reproducible, and systematic process.

With the rapidly changing landscape of therapeutic advances in cellular immunotherapies for MCL, the timing, sequence, and feasibility of these novel therapies represent a challenge. We envision that clinical trials using CAR T-cell therapies potentially in earlier lines of treatment, or in combination with BTKi, may emerge. As a result, treatment algorithms for this disease are likely to continue to evolve.
We hope these clinical practice recommendations will serve as a tool to guide clinicians managing patients with newly diagnosed and R/R MCL.

Author contributions Conception and design: PNM, TSF, BNS, SPR, MH. Financial support: N/A Collection and assembly of data: PNM, TSF, AK, and MH. Data analysis: AK. Interpretation: All authors. Manuscript writing: First draft prepared by PNM, TSF, and MH. All authors helped revising the manuscript. Final approval of manuscript: All authors.

\section{Compliance with ethical standards}

Conflict of interest MH reports: Research Support/Funding: Takeda Pharmaceutical Company; Spectrum Pharmaceuticals; Astellas Pharma. Consultancy: Janssen; Incyte Corporation; ADC Therapeutics; Celgene Corporation; Omeros, Verastem, MorphoSys. Speaker's Bureau: Sanofi Genzyme, AstraZeneca, BeiGene. TSF reports: Research Support/Funding: Novartis, Portola, Curis; Consultancy: Adaptive Biotechnologies, AbbVie, KaryoPharm, Kite, MorphoSys, Pharmacyclics, Sanofi. Speaker's Bureau: Sanofi, Seattle Genetics, AstraZeneca, Celgene/ Bristol-Myers Squibb, Adaptive Biotechnologies. PNM reports: Speaker's Bureau: Kite, a Gilead Company, Incyte. JBC reports: Consulting/Advisory Boards: Janssen, Adicet, BeiGene, Cellectar, Kite/Gilead, Adaptive, Aptitude Health, Astra Zeneca, Loxo. Research Support/Funding: Novartis, BMS/Celgene, Takeda, Genentech, Astra Zeneca, Loxo, LAM, Atara, BeiGene. NG reports: Speakers Bureau: Kite Pharma, Astra Zeneca, AbbVie, Janssen, BMS, Epizyme; Consulting/Advisory: Kite Pharma, Astra Zeneca, AbbVie, Janssen, BMS, Incyte, Epizyme, Karyopharma, Genmab, Adaptive Biotech, T.G. Therapeutics, ADC Therapeutics, BeiGene; Research funding: Genentech/Roche, BMS, T.G. Therapeutics, Pharmacyclics, Kite Pharma. MK reports: Research Support/ Funding: TG Therapeutics, Genentech. Consultancy: AbbVie, KaryoPharm, Kite, AstraZeneca, Celgene/ Bristol-Myers Squibb, Adaptive Biotechnologies, Curio Science. Speaker's bureau: SeaGen. MJ reports: Research Support/Funding: Janssen, Celgene, Abbvie, Gilead, Roche, Consultancy: Janssen; Incyte; Gilead, Roche, Bristol-Myers Squibb; Acerta; BioInvent. JWF reports: DSMB activities: Bayer, Ascerta, Novartis. CCS reports: Research support/Funding: ADC Therapeutics, Sanofi, Roche. Consulting/Advisory: Sanofi, ADC Therapeutics, Roche, Karyopharm Therapeutics, Celgene/BristolMyers Squibb, Incyte. Honoraria: Bristol-Myers Squibb, Janssen Oncology, AstraZeneca. Travel Grants: Roche, Janssen, Takeda, ADC Therapeutics. VB reports: Advisory Board: Kite, Gamida Cell: Research support/Funding: Incyte, Gamida Cell, GT Biopharma. TP reports: Research Support/Funding: Incyte, BMS/Celgene, Bayer, Abbvie, Genentech; Consultancy: BMS, Bayer, Abbvie, Genentech, Gilead, Gilead/Kite TG Therapeutics, ADC Therapeutics, Incyte, MrophoSys, BeiGene, AstraZenca, Epizyme. FLL reports: Research Support/Funding: Kite, a Gilead Company; Scientific Advisor: Allogene, Amgen, Bluebird Bio, BMS/Celgene, Calibr, Celgene, GammaDelta Therapeutics, Iovance, Kite/Gilead, Legend Biotech, Novartis, Wugen; Consultancy: Cellular Biomedicine Group, Cowen Consulting, Gerson Lehrman Group (GLG), EcoR1, Emerging Therapies. BK reports: Research Support/Funding: Abbvie, Acerta, AstraZeneca, Beigene, Celgene, Genentech; Consulting: Abbvie, Acerta, AstraZeneca, Beigene, Celgene, Kite, Genentech, Pharmacyclics, Janssen. MAKD reports: Consultancy: Pharmacyclics, Daiichi Sankyo. AFH reports: Consulting or Advisory Role: Bristol-Myers Squibb, Merck, Seattle Genetics, Karyopharm, Genentech/Roche; Research Funding: Bristol-Myers Squibb (Inst), Genentech/Roche (Inst), Merck (Inst), Seattle Genetics (Inst), ADC Therapeutics (Inst), Gilead/Kite Pharma (Inst); Travel, Accommodations, Expenses: 
Bristol-Myers Squibb. KJM reports: Research funding: Pharmacyclics, BMS, Merck, Novartis; Consultancy: Pharmacyclics, Janssen, Morphosys, Celgene, Beigene, Kite, Karyopharm, ADC Therapeutics, Seattle Genetics. VPK reports: Research funding: Novartis. DJI reports: Research funding: Acerta Pharma. CSS reports: Consultancy/ Advisory boards: Juno Therapeutics, Sanofi-Genzyme, Spectrum Pharmaceuticals, Novartis, Genmab, Precision Biosciences, Kite/a Gilead Company, Celgene/BMS, Gamida Cell, Karyopharm Therapeutics, GSK; Research Funding: Juno Therapeutics, Celgene/BMS, Bristol-Myers Squibb, Precision Biosciences and Sanofi-Genzyme. PD reports: consultancy for AbbVie, AstraZeneca, bluebird bio, Gilead, Janssen, Novartis, Riemser, Roche; speakers bureau for AbbVie, AstraZeneca, Gilead, Novartis, Riemser, Roche; research support from Riemser. JPL reports: Research support: Leukemia \& Lymphoma Society, Genentech Foundation. Consulting: Sutro, Miltenyi, AstraZeneca, Epizyme, BMS/Celgene, Regeneron, Bayer, Gilead/Kite, Karyopharm, GenMab, Genentech/Roche, Abbvie, Incyte. MD reports: institutional research funding: Abbvie, Bayer, Celgene, Janssen, Roche. Advisory Boards: Astra Zeneca, Bayer, Beigene, Celgene, Genmab, Gilead, Incyte, Janssen, Novartis, Roche. Speaker's honoraria: Amgen, Astra Zeneca, Bayer, Celgene, Gilead, Janssen, Roche. BTH reports: research funding: Kite/Gilead, Celgene/BMS, Genentech, Pharmacyclics, Abbvie. Consulting: Kite/Gilead, Celgene/BMS, Novartis, Beigene, AstraZeneca, Genentech, Abbvie.

Publisher's note Springer Nature remains neutral with regard to jurisdictional claims in published maps and institutional affiliations.

\section{References}

1. Swerdlow SH, Campo E, Harris NL, Jaffe ES, Pileri SA, Stein H, et al. WHO classification of tumours of haematopoietic and lymphoid tissues. WHO Classification of Tumours, Revised 4th Edition, Volume 2.2. p. 215-344.

2. Martin P, Chadburn A, Christos P, Weil K, Furman R, Ruan J, et al. Outcome of deferred initial therapy in mantle-cell lymphoma. J Clin Oncol. 2009;27:1209-13.

3. Dreyling M, Campo E, Hermine O, Jerkeman M, Le Gouill S, Rule S, et al. ESMO Guidelines Committee. Newly diagnosed and relapsed mantle cell lymphoma: ESMO Clinical Practice Guidelines for diagnosis, treatment and follow-up. Ann Oncol. 2017;28: iv62-iv71.

4. Robinson S, Dreger P, Caballero D, Corradini P, Geisler C, Ghielmini M, et al. European MCL Network and the Lymphoma Working Party of the European Society for Blood and Marrow Transplantation. The EBMT/EMCL consensus project on the role of autologous and allogeneic stem cell transplantation in mantle cell lymphoma. Leukemia. 2015;29:464-73.

5. Maddocks K. Update on mantle cell lymphoma. Blood. 2018;132:1647-56.

6. Eskelund CW, Dahl C, Hansen JW, Westman M, Kolstad A, Pedersen L, et al. TP53 mutations identify younger mantle cell lymphoma patients who do not benefit from intensive chemoimmunotherapy. Blood. 2017;130:1903-10. https://doi.org/10. 1182/blood-2017-04-779736.

7. Jerkeman M, Eskelund CW, Hutchings M, Räty R, Wader K, Laurellet A. al. Ibrutinib, lenalidomide, and rituximab in relapsed or refractory mantle cell lymphoma (PHILEMON): a multicentre, open-label, single-arm, phase 2 trial. Lancet Haematol. 2018;5: e109-e116.

8. Aukema SM, Hoster E, Rosenwald A, Canoni D, Delfau-Larue M, Rymkiewicz G, et al. Expression of TP53 is associated with outcome of MCL independent of MIPI and Ki-67 in trials of the European-MCL Network. Blood. 2018;131:417-20.
9. Wang ML, Rule S, Martin P, Goy A, Auer R, Kahl B, et al. Targeting BTK with ibrutinib in relapsed or refractory mantle-cell lymphoma. N. Engl J Med. 2013;369:507-16.

10. Wang ML, Munoz J, Goy A, Locke F, Jacobson C, Hill B, et al. KTE-X19 CAR T-cell therapy in relapsed or refractory mantlecell lymphoma. N. Engl J Med. 2020;382:1331-42.

11. Dreyling M, Lenz G, Hoster E, Gisselbrecht C, Schmits R, Metzneret B. al. Early consolidation by myeloablative radiochemotherapy followed by autologous stem cell transplantation in first remission significantly prolongs progression-free survival in mantle-cell lymphoma: results of a prospective randomized trial of the European MCL Network. Blood. 2005;105:2677-84.

12. Fenske TS, Zhang MJ, Carreras J, Ayala E, Burns L, Cashen A, et al. Autologous or reduced-intensity conditioning allogeneic hematopoietic cell transplantation for chemotherapy-sensitive mantle-cell lymphoma: analysis of transplantation timing and modality. J Clin Oncol. 2014;32:273-81.

13. Zoellner A, Unterhalt M, Stilgenbauer S, Hübel K, Thieblemont C, Metzner B, et al. Autologous stem cell transplantation in first remission significantly prolongs progression-free and overall survival in mantle cell lymphoma. Int Conf Malig Lymphoma, Hematological Oncol. 2019;37:43-44.

14. Gerson JN, Handorf E, Villa D, Gerrie A, Chapani P, Li S, et al. Survival outcomes of younger patients with mantle cell lymphoma treated in the rituximab era. J Clin Oncol. 2019;37:471-80.

15. LaCasce A, Vandergrift J, Rodriguez M, Abel G, Crosby A, Czuczman M, et al. Comparative outcome of initial therapy for younger patients with mantle cell lymphoma: an analysis from the NCCN NHL Database. Blood. 2012;119:2093-9.

16. Hamadani M, Saber W, Ahn K, Carreras J, Cairo M, Fenske T, et al. Allogeneic hematopoietic cell transplantation for chemotherapy-unresponsive mantle cell lymphoma: a cohort analysis from the center for international blood and marrow transplant research. Biol Blood Marrow Transpl. 2013;19:625-31. https://doi.org/10.1016/j.bbmt.2013.01.009.

17. Krüger WH, Hirt C, Basara N, Sayer H, Behre G, Fischer T, et al. Allogeneic stem cell transplantation for mantle cell lymphomafinal report from the prospective trials of the East German Study Group Haematology/Oncology (OSHO). Ann Hematol. 2014;93:1587-97.

18. Le Gouill S, Thieblemont C, Oberic L, Moreau A, Bouabdallah K, Dartigeas C, et al. Rituximab after autologous stem-cell transplantation in mantle-cell lymphoma. N. Engl J Med. 2017;377:1250-60.

19. Owen C, Berinstein NL, Christofides A, Sehn L. Review of Bruton tyrosine kinase inhibitors for the treatment of relapsed or refractory mantle cell lymphoma. Curr Oncol. 2019;26: e233-e240. https://doi.org/10.3747/co.26.4345.

20. Loblaw DA, Prestrud A, Somerfield M, Oliver T, Brouwers M, Nam R, et al. American Society of Clinical Oncology Clinical Practice Guidelines: formal systematic review-based consensus methodology. Clin Oncol. 2012;30:3136-40.

21. Murphy MK, Black NA, Lamping DL, McKee CM, Sanderson $\mathrm{CF}$, Askham J, et al. Consensus development methods, and their use in clinical guideline development. Health Technol Assess. 1998;2:i-iv.

22. Ramakrishna N, Temin S, Chandarlapaty S, Crews J, Davidson N, Esteva $\mathrm{F}$, et al. Recommendations on disease management for patients with advanced human epidermal growth factor receptor 2-positive breast cancer and brain metastases: American society of clinical oncology clinical practice guideline. J Clin Oncol. 2014;32:12100-8. https://doi.org/10.1200/JCO.2013.54.0955.

23. Berkman ND, Lohr KN, Ansari MT, Balk E, Kane R, McDonagh MS, et al. Grading the strength of a body of evidence when assessing health care interventions: An EPC update. J Clin 
Epidemiol. 2015;68:1312-24. https://doi.org/10.1016/j.jclinepi. 2014.11.023.

24. Geisler CH, Kolstad A, Laurell A, Jerkeman M, Raty R, Andersen $\mathrm{N}$, et al. Nordic MCL2 trial update: six-year follow-up after intensive immunochemotherapy for untreated mantle cell lymphoma followed by BEAM or BEAC + autologous stem-cell support: still very long survival but late relapses do occur. Br J Haematol. 2012;158:355-62. https://doi.org/10.1111/j.1365-2141. 2012.09174.x.

25. Evens AM, Winter JN, Hou N, Nelson B, Rademaker A, Patton D, et al. A phase II clinical trial of intensive chemotherapy followed by consolidative stem cell transplant: Long-term follow-up in newly diagnosed mantle cell lymphoma. $\mathrm{Br} \mathrm{J}$ Haematol. 2008;140:385-93.

26. Hermine O, Hoster E, Walewski J, Bosly A, Stilgenbauer S, Thieblemont $\mathrm{C}$, et al. Addition of high-dose cytarabine to immunochemotherapy before autologous stem-cell transplantation in patients aged 65 years or younger with mantle cell lymphoma (MCL Younger): a randomised, open-label, phase 3 trial of the European Mantle Cell Lymphoma Network. Lancet. 2016;388:565-75.

27. Chhabra S, Thapa B, Szabo A, Konings S, D’Souza A, Dhakal B, et al. Utilization and cost implications of hematopoietic progenitor cells stored for a future salvage autologous transplantation or stem cell boost in myeloma patients. Biol Blood Marrow Transpl. 2020;26:2011-7. https://doi.org/10.1016/j.bbmt.2020.07.019.

28. Damon LE, Johnson JL, Niedzwiecki D, Cheson B, Hurd D, Bartlett N. et al. Immunochemotherapy and autologous stem-cell transplantation for untreated patients with mantle cell lymphoma: CALGB 59909. J Clin Oncol. 2009;27:6101-8. https://doi.org/10. 1200/JCO.2009.22.2554.
29. Hoster E, Klapper W, Hermine O, Kluin-Nelemans H, Walewski J, van Hoof $\mathrm{A}$, et al. Confirmation of the mantle-cell lymphoma international prognostic index in randomized trials of the European mantle-cell lymphoma network. J Clin Oncol. 2014;32:1338-46.

30. Lin RJ, Ho C, Hilden PD, Barker J, Giralt S, Hamlin P, et al. Allogeneic haematopoietic cell transplantation impacts on outcomes of mantle cell lymphoma with TP53 alterations. Br J Haematol. 2019;184:1006-10.

31. Dreyling M, Jurczak W, Jerkeman M, Silva R, Rusconi C, Trneny $\mathrm{M}$, et al. Ibrutinib versus temsirolimus in patients with relapsed or refractory mantle-cell lymphoma: an international, randomised, open-label, phase 3 study. Lancet. 2016;387:770-8.

32. Khouri IF, Lee MS, Saliba RM, Jun G, Fayad L, Younes A, et al. Nonablative allogeneic stem-cell transplantation for advanced/ recurrent mantle-cell lymphoma. J Clin Oncol. 2003;21:4407-12. https://doi.org/10.1200/JCO.2003.05.501.

33. Maris MB, Sandmaier BM, Storer BE, Chauncey T, Stuart M, Maziarz R, et al. Allogeneic hematopoietic cell transplantation after fludarabine and 2 Gy total body irradiation for relapsed and refractory mantle cell lymphoma. Blood. 2004;104:3535-42. https://doi.org/10.1182/blood-2004-06-2275.

34. Cruz JG, Martino R, Balsalobre P, Heras I, Pinana J, Serrano D, et al. Long-TErm Results of Fludarabine/melphalan as A Reduced-intensity Conditioning Regimen in Mantle Cell Lymphoma: the GELTAMO experience. Ther Adv Hematol. 2011;2:5-10. https://doi.org/10.1177/2040620710396752.

35. Kanate AS, Kumar A, Dreger P, Dreyling M, Le Gouill S, Corradini $\mathrm{P}$, et al. Maintenance Therapies for Hodgkin and NonHodgkin Lymphomas After Autologous Transplantation: A Consensus Project of ASBMT, CIBMTR, and the Lymphoma Working Party of EBMT. JAMA Oncol. 2019;5:715-22. https:// doi.org/10.1001/jamaoncol.2018.6278.

\section{Affiliations}

\section{Pashna N. Munshi ${ }^{1}{ }^{1} \cdot$ Mehdi Hamadani ${ }^{2}{ }^{2} \cdot$ Ambuj Kumar $^{3}{ }^{3} \cdot$ Peter Dreger $\mathbb{D}^{4} \cdot$ Jonathan W. Friedberg ${ }^{5} \cdot$

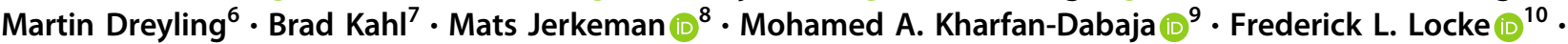 Mazyar Shadman ${ }^{11}$ - Brian T. Hill ${ }^{12}$ - Sairah Ahmed ${ }^{13}$. Alex F. Herrera ${ }^{14} \cdot$ Craig S. Sauter $^{15}$. Veronika Bachanova (D) ${ }^{16} \cdot$ Nilanjan Ghosh ${ }^{17} \cdot$ Matthew Lunning $^{18} \cdot$ Vaishalee P. Kenkre $^{19} \cdot$ Mahmoud Aljurf $^{20}$. Michael Wang $\mathbb{D}^{21} \cdot$ Kami J. Maddocks ${ }^{22} \cdot$ John P. Leonard ${ }^{23} \cdot$ Manali Kamdar $^{24} \cdot$ Tycel Phillips $^{25}$. Amanda F. Cashen ${ }^{26}$. David J. Inwards ${ }^{27}$. Anna Sureda ${ }^{28}$. Jonathon B. Cohen ${ }^{29} \cdot$ Sonali M. Smith $^{30}$. Carmello Carlo-Stella $\mathbb{I D}^{31} \cdot$ Bipin Savani ${ }^{32} \cdot$ Stephen P. Robinson ${ }^{33} \cdot$ Timothy S. Fenske $^{34}$}

1 MedStar Georgetown University Hospital, Washington, DC, USA

2 CIBMTR \& BMT \& Cellular Therapy Program, Medical College of Wisconsin, Milwaukee, WI, USA

3 Department of Internal Medicine, Office of Research, Morsani College of Medicine, University of South Florida, Tampa, FL, USA

4 University of Heidelberg, Heidelberg, Germany

5 Wilmot Cancer Institute, University of Rochester, Rochester, NY, USA

6 Department of Medicine III, LMU Hospital Munich, Munich, Germany

7 Washington University School of Medicine, St. Louis, MO, USA
8 Department of Oncology, Skane University Hospital, Lund University, Lund, Sweden

9 Division of Hematology-Oncology and Blood and Marrow Transplantation and Cellular Therapies Program, Mayo Clinic, Jacksonville, FL, USA

10 Department of Blood and Marrow Transplant and Cellular Immunotherapy, Moffitt Cancer Center, Tampa, FL, USA

11 Fred Hutchinson Cancer Research Center and University of Washington, Seattle, WA, USA

12 Department of Hematology and Medical Oncology, Taussig Cancer Institute, Cleveland Clinic Foundation, Cleveland, OH, USA

13 Department of Lymphoma, Myeloma and Stem Cell Transplantation \& Cellular Therapy, University of Texas MD Anderson Cancer Center, Houston, TX, USA 
14 Department of Hematology and Hematopoietic Cell Transplantation, City of Hope Medical Center, Duarte, CA, USA

15 Department of Medicine, Memorial Sloan Kettering Cancer Center and Weill Cornell Medical College, New York, NY, USA

16

Division of Hematology, Oncology and Transplantation, University of Minnesota, Minneapolis, MN, USA

17 Levine Cancer Institute, Atrium Health, Charlotte, NC, USA

18 University of Nebraska Medical Center, Omaha, NE, USA

19 University of Wisconsin, Division of Hematology and Oncology, Madison, WI, USA

20 King Faisal Specialist Hospital, Riyadh, Saudi Arabia

21 Department of Lymphoma and Myeloma, University of Texas M. D. Anderson Cancer Center, Houston, TX, USA

Division of Hematology, Ohio State University, Columbus, $\mathrm{OH}$, USA

Weill Cornell Medicine, New York, NY, USA

24

Division of Hematology, Hematologic Malignancies and Stem Cell Transplantation, University of Colorado Cancer Center, Denver, CO, USA
25 Rogel Cancer Center, University of Michigan, Ann Arbor, MI, USA

26 Division of Oncology, Section of Stem Cell Transplantation, Washington University School of Medicine, St Louis, MO, USA

27 Division of Hematology, Mayo Clinic Rochester, Rochester, MN, USA

28 Clinical Hematology Department, Catalan Institute of Oncology, Institut d'Investigació Biomèdica de Bellvitge IDIBELL, Universitat de Barcelona, Barcelona, Spain

29 Winship Cancer Institute, Atlanta, GA, USA

30 Section of Hematology/Oncology, University of Chicago, Chicago, IL, USA

31 Department of Biomedical Sciences, Humanitas University and IRCCS Humanitas Research Hospital, Milan, Italy

32 Vanderbilt University Medical Center, Nashville, TN, USA

33 University Hospital Bristol NHS Foundation Trust, London, UK

34 Department of Medicine, Medical College of Wisconsin, Milwaukee, WI, USA 